\title{
Frecuencia y Susceptibilidad Antimicrobiana de Bacterias Causantes de Mastitis en Bovinos de un Establo de Trujillo, Perú
}

\author{
Frequency and Antimicrobial Susceptibility of Bacteria Causing Mastitis in a \\ Dairy Farm in Trujillo, Peru
}

\author{
Roxana Rodríguez Pérez ${ }^{1}$, Eduardo Muñoz Ganoza ${ }^{2,3}$
}

\section{Resumen}

\begin{abstract}
Se determinó la frecuencia y susceptibilidad antimicrobiana de bacterias causantes de mastitis en bovinos de un establo del distrito de Conache, Trujillo, Perú, entre septiembre y diciembre de 2015. Se recolectaron muestras de leche de 140 cuartos individuales de 35 vacas que se les hizo el control de mastitis mediante observación directa y taza de fondo negro. Para el análisis microbiológico se sembraron las muestras en agar sangre y agar Mac Conkey y se incubaron a $37^{\circ} \mathrm{C}$ por $24 \mathrm{~h}$. Para determinar las bacterias gram positivas se realizaron pruebas de catalasa, coagulasa, fermentación de manitol y hemólisis; y para las gram negativas se realizaron pruebas de agar-hierro-triple azúcar (TSI), agar lisina-hierro (LIA), citrato de Simmons, ureasa, formación de indol, rojo metilo, Voges Proskauer y caldo glutamato. La susceptibilidad antimicrobiana se determinó mediante el método de Kirby-Bauer con discos de ampicilina, clindamicina, doxiciclina, eritromicina, estreptomicina, gentamicina, oxacilina y rifampicina. Se detectaron 31 vacas con mastitis bacteriana, donde el $76 \%$ presentó bacterias gram negativas y $24 \%$ gram positivas. Las bacterias gram negativas más frecuentes fueron E. coli $(28 \%)$ y Klebsiella sp. (24\%), y la bacteria gram positiva más frecuente fue Staphylococcus aureus (16\%). Pseudomonas aeruginosa fue resistente a la mayoría de antibióticos, excepto a la eritromicina (susceptibilidad intermedia). E. coli presentó susceptibilidad intermedia a la eritromicina y resistencia a la oxacilina y rifampicina. Staphylococvcus aureus presentó susceptibilidad intermedia a la clindamicina y eritromicina y resistencia a la ampicilina.
\end{abstract}

Palabras clave: mastitis bovina; susceptibilidad antimicrobiana; bacterias gramnegativas; bacterias grampositivas

\footnotetext{
${ }^{1}$ Escuela de Microbiología y Parasitología, ${ }^{2}$ Departamento de Microbiología y Parasitología, Facultad de Ciencias Biológicas, Universidad Nacional de Trujillo, Perú

${ }^{3}$ E-mail: eferzo@yahoo.es
}

Recibido: 11 de enero de 2017

Aceptado para publicación: 14 de julio de 2017 
The frequency and antimicrobial susceptibility of mastitis caused by bacteria in cattle from a dairy farm in the Conache district, Trujillo, Peru, between September and December 2015 were determined. Milk samples were collected from 140 quarters of 35 cows that were checked for mastitis by direct observation in a cup with black background. For the microbiological analysis, the samples were cultured in blood agar and MacConkey agar and incubated at $37^{\circ} \mathrm{C}$ for $24 \mathrm{~h}$. To determine gram-positive bacteria tests of catalase, coagulase, mannitol fermentation and hemolysis were performed, and for gram-negative triple sugar iron agar (TSI), lysine-iron agar (LIA), Simmons citrate, urease, indole, methyl red, Voges Proskauer and glutamate broth were used. Antimicrobial susceptibility was determined by the Kirby-Bauer method with ampicillin, clindamycin, doxycycline, erythromycin, streptomycin, gentamicin, oxacillin and rifampicin disks. Thirty one cows with bacterial mastitis were detected, where $76 \%$ had gram-negative bacteria and $24 \%$ had gram-positive bacteria. The most frequent gram-negative bacteria were $E$. coli $(28 \%)$ and Klebsiella sp. (24\%), and the most frequent gram-positive bacteria was Staphylococcus aureus (16\%). Pseudomonas aeruginosa was resistant to most antibiotics except erythromycin (intermediate susceptibility). E. coli presented intermediate susceptibility to erythromycin and resistance to oxacillin and rifampicin. Staphylococcus aureus presented intermediate susceptibility to clindamycin and erythromycin and resistance to ampicillin.

Key words: bovine mastitis; antimicrobial susceptibility; gram-negative bacteria; grampositive bacteria

\section{INTRODUCCIÓN}

La mastitis es la inflamación de la glándula mamaria, usualmente inducida por una infección bacteriana intramamaria que, además, puede ser causada por agentes fúngicos, virales o por daños de tipo traumático, térmico o químico (Gonzales, 1996; Karina, 2011). La respuesta inflamatoria intenta destruir o neutralizar el agente ofensivo, reparar los tejidos dañados y retornar la glándula a su función normal (Figueroa, 2008).

La gran diversidad de bacterias causantes de mastitis bovina se debe a las características epidemiológicas y ambientales de los microorganismos contagiosos. Entre los microorganismos más frecuentes se encuentran Streptococcus (Strep) agalactiae, Staphylococcus (Staph) aureus, Staph. hyicus, Mycoplasma sp, Staph. coagulasa negativos (SCN), Corynebacterium bovis, Escherichia coli, Klebsiella sp, Pseudomonas sp, Strep uberis, Strep. dysgalactiae y Enterococcus sp (Ferguson et al., 2007).

En el manejo integral de la mastitis se incluye el uso de antimicrobianos administrados por vía sistémica e intramamaria. Dentro de los grupos farmacológicos más utilizados destacan los betalactámicos (penicilinas, cefalosporinas), nuevos betalactámicos, macrólidos y algunos aminoglicósidos (Martínez et al., 2013). Asimismo, algunos de estos antimicrobianos también se utilizan con fines profilácticos durante el periodo de secado para reducir la mastitis en la siguiente lactancia (Martínez et al., 2013). La susceptibilidad de los microorganismos frente a los antimicrobianos varía de una región a otra, lo que obliga a los investigadores a realizar estudios epidemiológicos para establecer la 
presencia de la enfermedad y sus agentes causales en cada región, así como realizar estudios in vitro de manera periódica para determinar la sensibilidad y el estado de resistencia (Martínez et al., 2013).

Una de las principales actividades económicas de los habitantes de los valles en los Andes peruanos es la crianza de ganado vacuno con fines de comercialización de la leche; sin embargo, uno de los problemas de esta crianza es la mastitis, que ocasiona la disminución de la calidad y cantidad de leche $\mathrm{y}$, con ello, severas pérdidas económicas (Santiváñez et al., 2013).

En los últimos tres años, la mastitis en el Perú arroja pérdidas económicas de aproximadamente US\$ 2500 000, pero este monto solo incluye a la mastitis clínica, la cual representa el $20-30 \%$ de la mastitis (SENASA, 2015). Según estimaciones del Ministerio de Agricultura y Ganadería, las pérdidas económicas de la región La Libertad varían entre 25 y $32 \%$ debido al deterioro de la calidad de la leche proveniente de vacas con mastitis (SENASA, 2015).

El objetivo del presente estudio fue determinar la frecuencia y susceptibilidad antimicrobiana de bacterias causantes de mastitis en bovinos de un establo de Conache, del distrito de Laredo, Trujillo, Perú.

\section{Materiales y Métodos}

\section{Lugar de Estudio y Animales}

El estudio se realizó en un establo de la localidad de Conache, en el distrito de Laredo, Trujillo (Perú), porque abastece de leche a las localidades cercanas y se desconoce, epidemiológicamente, el curso de la mastitis bovina en la región. El establo tenía 57 vacas en producción y no disponía de un médico veterinario permanente que planifique y ejecute programas de educación sanitaria para el personal del establo y muestreos microbiológicos periódicos, ni se tenía la posibilidad de supervisar el diagnóstico y tratamiento de vacas con mastitis.

El tamaño de muestra se determinó mediante la fórmula del tamaño mínimo muestreal $(n=35)$, utilizando un muestreo probabilístico aleatorio simple, tomando en consideración el número total de vacas del establo $(\mathrm{n}=65)$, un nivel de confianza de $95 \%$ y un error máximo admisible de 5\% (Hernández et al., 2003).

\section{Muestras de Leche}

Las muestras de leche $(n=140)$ se tomaron entre septiembre y diciembre de 2015, mediante ordeño manual de los cuatro cuartos de 35 vacas elegidas al azar. Los animales eran Holstein y mestizos.

Previo a la toma de las muestras, se realizaron exámenes clínicos a las vacas seleccionadas para determinar posibles signos de mastitis; además se realizó la prueba de la taza de fondo negro (Bedolla et al., 2007; Ramírez et al., 2011). Para la toma de las muestras, cada pezón fue sumergido en una solución germicida durante 30 segundos y secado con papel toalla; luego, se desinfectó el extremo de cada pezón con algodón humedecido en alcohol etílico al 70\% (Agencia Agraria Trujillo, 2010; Nieto et al., 2012), se extrajeron $10 \mathrm{ml}$ de leche de cada cuarto en frascos estériles de polietileno con tapa rosca y, previa identificación, se llevaron al Laboratorio de Inmunología de la Facultad de Ciencias Biológicas de la Universidad Nacional de Trujillo en un cooler con gel refrigerante a $4{ }^{\circ} \mathrm{C}$. Las muestras fueron sembradas dentro de las dos horas de su colección.

\section{Cultivo Microbiológico y Pruebas Bio- químicas}

Las muestras fueron sembradas por estría en cuatro cuadrantes en agar sangre y agar Mac Conkey e incubadas a $37^{\circ} \mathrm{C}$ por 24-48 h. Se observó la morfología de las co- 
lonias y se aislaron en cultivo puro para su identificación. A cada cultivo se le realizó la tinción de Gram para confirmar su pureza y diferenciar a las bacterias gram positivas de las gram negativas (Amand de Mendieta et al., 2001).

Se utilizó la prueba de catalasa en los cocos gram positivos para diferenciar Staphylococcus de Streptococcus. La prueba de coagulasa en tubo diferenció Staph. aureus y otros Staphylococcus coagulasa positiva (SCP) de los SCN. La prueba de fermentación de manitol en agar manitol salado diferenció a las bacterias del género Staphylococcus. Además, los cocos gram positivos fueron sembrados en agar sangre, donde se diferenció el tipo de hemólisis de las colonias (Mac Faddin, 2000; Sacsaquispe y Ventura, 2001).

Se hicieron pruebas para bioquímica diferencial en agar-hierro-triple azúcar (TSI) a las colonias de bacilos gram negativos para identificar bacterias fermentadoras de glucosa, lactosa o sacarosa. Asimismo, se observó la producción de gas $\left(\mathrm{H}_{2} \mathrm{~S}\right)$. En la prueba de agar hierro-lisina (LIA) se observó la decarboxilación de lisina o desaminación de lisina y la producción de $\mathrm{H}_{2} \mathrm{~S}$. Además, se realizaron las pruebas de citrato de Simmons, hidrólisis de urea, producción de indol, rojo de metilo, Voges Proskauer y caldo glutamato (Mac Faddin, 2000; Sacsaquispe y Ventura, 2001).

\section{Susceptibilidad Antimicrobiana}

De cada bacteria identificada se prepararon suspensiones equivalentes a una concentración de $1.5 \times 10^{8} \mathrm{UFC} / \mathrm{ml}$ comparado con el tubo 0.5 del tubo de nefelómetro de McFarland (Calvinho et al., 1991). Se inoculó la suspensión en placas petri con agar Mueller-Hinton, de manera que cubra toda la superficie y se dejó secar a temperatura ambiente durante 3 a $5 \mathrm{~min}$. Luego se aplicaron por separado los discos de ampicilina $(10 \mu \mathrm{g})$, clindamicina $(2 \mu \mathrm{g})$, doxiciclina $(30 \mu \mathrm{g})$, eritromicina $(15 \mu \mathrm{g})$, estreptomicina $(10 \mu \mathrm{g})$, gentamicina (10 ìg), oxacilina $(1 \mu \mathrm{g})$ y rifampicina $(5 \mu \mathrm{g})$. Se incubó a $37^{\circ} \mathrm{C}$ por 24 h y se observó la presencia de un halo de inhibición alrededor de los discos y se midió su diámetro. Los resultados fueron interpretados de acuerdo con los criterios del Clinical Laboratory Standards Institute (NCCLS, 2002).

\section{Análisis Estadístico}

Debido al carácter cualitativo de las variables de este estudio, el análisis de los resultados se realizó mediante estadística descriptiva. Las variables que se establecieron fueron la frecuencia de bacterias identificadas en las muestras de leche y la susceptibilidad antimicrobiana.

\section{Resultados y Discusión}

De las 35 vacas que fueron muestreadas al azar, 31 vacas (89\%) presentaron mastitis. Esta es una elevada incidencia de mastitis en un establo lechero. Armenteros et al. (2002) refieren haber encontrado el $3 \%$ de cuartos mamarios con mastitis clínica en Cuba.

De las 31 vacas con mastitis, $25(81 \%)$ presentaron mastitis clínica diagnosticada con la prueba de la taza de fondo negro. De los 124 cuartos de las vacas positivas a mastitis, 123 cuartos (99\%) presentaron mastitis, encontrándose solo un cuarto sano. La elevada tasa de mastitis clínica estaría fundamentada por la falta de prácticas de control de mastitis, el estrés sufrido por los animales expuestos a las lluvias en el verano, y la presencia de insectos, aves de corral y perros. En EEUU, la tasa de incidencia de mastitis clínica por lactación es de $14 \%$, donde los costos varían entre US\$ 108 y 122 por caso, considerando los medicamentos, el médico veterinario, las medidas preventivas, el desecho y las pérdidas de leche (Heringstad et al., 2000). El costo atribuible a las formas subclínicas de la mastitis asciende a la mayor parte del costo total de la industria que previene la mastitis, entre US\$ 100 y 150 por vaca/año o del 50 al 
Cuadro 1. Frecuencia de bacterias presentes en muestras de leche de 31 vacas con mastitis en un establo lechero de Conache (Laredo, Trujillo) (septiembre-diciembre de 2015)

\begin{tabular}{llcc}
\hline Bacterias & & Número & Porcentaje \\
\hline Gram positivas & Staphylococcus aureus & 30 & 16 \\
& Streptococcus sp & 16 & 8 \\
Gram negativas & Escherichia coli & 54 & 28 \\
& Klebsiella sp & 46 & 24 \\
& Pseudomonas aeruginosa & 22 & 12 \\
& Shigella sp & 10 & 5 \\
& Proteus sp & 6 & 3 \\
& Enterobacter sp & 4 & 2 \\
& Citrobacter $\mathrm{sp}$ & 2 & 1 \\
\hline Total & & 190 & 100 \\
\hline
\end{tabular}

Cuadro 2. Susceptibilidad antimicrobiana de las bacterias asiladas en muestras de leche de 123 cuartos mamarios infectados de 31 vacas con mastitis. Interpretación de los diámetros de halos de inhibición ${ }^{\mathrm{A}}$ según el Clinical Laboratory Standards Institute (CLSI, 2015)

\begin{tabular}{lcccccccc}
\hline \multirow{2}{*}{ Bacterias } & \multicolumn{7}{c}{ Antibióticos } \\
\cline { 2 - 8 } & $\mathrm{Amp}^{1}$ & $\mathrm{Cc}^{2}$ & $\mathrm{Dxs}^{3}$ & $\mathrm{E}^{4}$ & $\mathrm{~S}^{5}$ & $\mathrm{Ge}^{6}$ & $\mathrm{Ox}^{7}$ & $\mathrm{R}^{8}$ \\
\hline Gram positivas & & & & & & & & \\
$\quad$ Staphylococcus aureus & $\mathrm{R}$ & $\mathrm{I}$ & $\mathrm{S}$ & $\mathrm{S}$ & $\mathrm{I}$ & $\mathrm{S}$ & $\mathrm{S}$ & $\mathrm{S}$ \\
$\quad$ Streptococcus $\mathrm{sp}$ & $\mathrm{S}$ & $\mathrm{S}$ & $\mathrm{I}$ & $\mathrm{S}$ & $\mathrm{R}$ & $\mathrm{R}$ & $\mathrm{S}$ & $\mathrm{S}$ \\
Gram negativas & & & & & & & & \\
$\quad$ Escherichia coli & $\mathrm{S}$ & $\mathrm{S}$ & $\mathrm{S}$ & $\mathrm{I}$ & $\mathrm{S}$ & $\mathrm{S}$ & $\mathrm{R}$ & $\mathrm{R}$ \\
Klebsiella $\mathrm{sp}$ & $\mathrm{S}$ & $\mathrm{I}$ & $\mathrm{S}$ & $\mathrm{R}$ & $\mathrm{I}$ & $\mathrm{S}$ & $\mathrm{R}$ & $\mathrm{R}$ \\
Pseudomonas aeruginosa & $\mathrm{R}$ & $\mathrm{R}$ & $\mathrm{R}$ & $\mathrm{I}$ & $\mathrm{R}$ & $\mathrm{R}$ & $\mathrm{R}$ & $\mathrm{R}$ \\
Shigella $\mathrm{sp}$ & $\mathrm{S}$ & $\mathrm{S}$ & $\mathrm{S}$ & $\mathrm{I}$ & $\mathrm{R}$ & $\mathrm{R}$ & $\mathrm{R}$ & $\mathrm{R}$ \\
$\quad$ Proteus $\mathrm{sp}$ & $\mathrm{S}$ & $\mathrm{S}$ & $\mathrm{S}$ & $\mathrm{I}$ & $\mathrm{I}$ & $\mathrm{S}$ & $\mathrm{R}$ & $\mathrm{R}$ \\
$\quad$ Enterobacter $\mathrm{sp}$ & $\mathrm{S}$ & $\mathrm{S}$ & $\mathrm{S}$ & $\mathrm{I}$ & $\mathrm{S}$ & $\mathrm{S}$ & $\mathrm{R}$ & $\mathrm{R}$ \\
Citrobacter $\mathrm{sp}$ & $\mathrm{S}$ & $\mathrm{S}$ & $\mathrm{S}$ & $\mathrm{I}$ & $\mathrm{R}$ & $\mathrm{S}$ & $\mathrm{R}$ & $\mathrm{R}$ \\
\hline
\end{tabular}

${ }^{1}$ ampicilina $10 \mu \mathrm{g} ;{ }^{2}$ clindamicinana $2 \mu \mathrm{g} ;{ }^{3}$ doxiciclina $30 \mu \mathrm{g} ;{ }^{4}$ eritromicina $15 \mu \mathrm{g} ;{ }^{5}$ estreptomicina 10 $\mu \mathrm{g} ;{ }^{6}$ gentamicina $10 \mu \mathrm{g} ;{ }^{7}$ oxacilina $1 \mu \mathrm{g} ;{ }^{8}$ rifampicina $5 \mu \mathrm{g}$

${ }^{A}$ R: Resistente; I: Intermedio; S: Susceptible 
$80 \%$ de las pérdidas de producción total (Burvenich et al., 2005).

En el Cuadro 1 se muestran las bacterias halladas y su frecuencia. Las bacterias aisladas con mayor frecuencia fueron las gram negativas $76 \%$ (144), y dentro de estas, las más representativas fueron $E$. coli (28\%) y Klebsiella sp (24\%). Sin embargo, Ruiz et al. (2011) reportan con mayor incidencia Staphylococcus sp (29.6\%) y Streptococcus sp (14.6\%), y con menor incidencia E. coli (2.1\%) y otros bacilos gram negativos $(1.4 \%)$, resultados obtenidos de 11 propiedades productoras de leche bovina del establo de Pernambuco, Brasil.

En este estudio se encontró una elevada frecuencia de enterobacterias, lo cual fue el reflejo de las condiciones del establo de Conache. En este establo, el ordeño es manual y no han implementado una sala de ordeño. Tampoco se realiza la limpieza de los utensilios ni se dispone de instalaciones apropiadas de agua, y los restos de leche se vierten en el suelo sin cuidado alguno.

Los resultados de la susceptibilidad antimicrobiana se muestran en el Cuadro 2. $P$. aeruginosa fue resistente a la mayoría de antibióticos, con excepción de la eritromicina, ante la cual presentó susceptibilidad intermedia. La mayor resistencia de las bacterias aisladas fue contra oxacilina y rifampicina, donde siete bacterias gram negativas mostraron resistencia. Por otro lado, Staph. aureus y Streptococcus sp fueron susceptibles a eritromicina, oxacilina y rifampicina.

Los resultados de este estudio coinciden con los de Alcántara y Uscátegui (2013), quienes reportaron que Staph. aureus manifestó resistencia frente a la ampicilina y sensibilidad a la eritromicina y gentamicina. No obstante, es de esperar que los resultados varíen entre regiones, dependiendo del tipo, frecuencia y modo de uso de los antibióticos. Así, por ejemplo, Faría et al. (2005) encontró alta resistencia de Staph. aureus y Streptococcus sp a los antimicrobianos.
La susceptibilidad antimicrobiana determinada in vitro ha sido considerada como un requisito previo para el tratamiento. Sin embargo, la actividad in vitro no garantiza la eficacia in vivo durante el tratamiento de la mastitis bovina (Pyörälä, 2009). La resistencia bacteriana trae como consecuencia la disminución en la respuesta al tratamiento, en caso de mastitis clínica, y la transmisión de bacterias resistentes a los consumidores a través de la cadena alimentaria, más aún cuando se consumen productos elaborados a partir de leche cruda (Tikofsky et al., 2003).

La ruta más común para la administración de antimicrobianos en los casos de mastitis es la vía intramamaria (IMM). Asimismo, se ha sugerido que la vía sistémica de administración es más eficiente que la IMM para el tratamiento de la mastitis clínica, ya que los antimicrobianos tendrían una mayor penetración en el tejido mamario (Ziv, 1980; Erskine, 2003), especialmente en casos de mastitis debido a bacterias coliformes (Wenz et al., 2001).

El tratamiento de la mastitis subclínica con antimicrobianos, en general, no es económico durante la lactancia debido a los altos costos del tratamiento y a su reducida eficacia. En un estudio con una gran cantidad de casos de mastitis subclínica, la tasa global de curación bacteriológica para el tratamiento antimicrobiano fue de $75 \%$ y la tasa sin tratamiento fue de $68 \%$ (Gentilini et al., 2000).

\section{Conclusiones}

- Las bacterias gram negativas fueron la causa más frecuente de mastitis en bovinos de un establo de Conache del distrito de Laredo (Trujillo).

- Las bacterias gram negativas más frecuentes fueron E. coli y Klebsiella sp y las gram positiva más frecuente fue Staphylococcus aureus.

- La bacteria más resistente a la acción antimicrobiana in vitro fue Pseudomonas aeruginosa. 


\section{Literatura Citada}

1. Agencia Agraria Trujillo. 2010. Mejoramiento del manejo de ganado vacuno lechero. Boletín Informativo de la Gerencia Regional de Agricultura. La Libertad: Gobierno Regional de La Libertad.

2. Alcántara Acosta L, Uzcátegui D. 2013. Epidemiologia de la mastitis bovina en la unidad de producción «La Chiquinquirá»». Tesis de grado. Zulia, Venezuela: Univ Nacional Experimental Sur del Lago. 85 p.

3. Amand de Mendieta V, Micheo C, Soriano $C$, Tabera A, Stefano A, Casasnovas G, et al. 2001. Aislamiento e identificación de patógenos mamarios de animales bovinos lecheros de la Cuenca Mar y Sierras. Vet Arg 18: 499-504.

4. Armenteros M, Peña J, Pulido JL, Linares E. 2002. Caracterización de la situación de la mastitis bovina en rebaños de lechería especializada en Cuba. Rev Salud Anim 24: 99-105.

5. Bedolla CC, Castañeda VH, Wolter W. 2007. Métodos de detección de la mastitis bovina. REDVET 8(9): 16957504. [Internet]. Disponible en: http:// www.veterinaria.org/revistas/redvet/ n090907/090702.pdf

6. Burvenich C, Monfardini J, Mehrzad A, Capuco V, Paape MJ. 2005. El papel de los leucocitos polimorfonucleares neutrófilos en la mastitis coniforme en bovinos. 23-26.

7. Calvinho LF, Delgado AR, Vitulich CA, Occhi HL, Canavesio VR, Zurbriggen MA, Tarabla HD. 1991. Susceptibilidad in vitro a los antimicrobianos de microorganismos aislados a partir de mastitis clínicas en tambos de la cuenca lechera santafesina. Vet Arg 8: 677-680.

8. [CLSI] Clinical Laboratory Standards Institute. 2015. Performance standards for antimicrobial disk and dilution susceptibility tests for bacteria isolated from animals. Approved Standards. $15^{\text {th }}$ ed. CLSI 35(1). [Internet]. Disponible en:
http://shop.clsi.org/site/Sample_pdf/ M02A12_sample.pdf

9. Erskine RJ. 2003. Antibacterial therapy of clinical mastitis - Part I. Drug selection. Part II Administration. North Am Vet Conf Proc. p 13-16.

10. Faría J, Valero K, D'Pool G, García A, Allara M. 2005. Sensibilidad a los agentes antimicrobianos de algunos patógenos mastitogénicos aislados de leche de cuartos de bovinos mestizos doble propósito. Rev Cient FCV-LUZ 15: 227-234.

11. Ferguson J, Azzaro G, Gambina M, Licitra G. 2007. Prevalence of mastitis pathogens in Ragusa, Sicily, from 2000 to 2006. J Dairy Sci 90: 5798-5813. doi: 10.3168/jds.2006-903

12. Figueroa PG, Ignacio J, Bedolla C, Carlos JL. 2008. Determinación de la prevalencia de mastitis bovina en el municipio de Tarímbaro, Michoacán, mediante la prueba de California. REDVET 9(10). [Internet]. Disponible en: http:// www.veterinaria.org/revistas/redvet/ n101008/101004.pdf

13. Gentilini E, Denamiel G, Llorente P, Godaly S, Rebuelto M, Degregorio $O$. 2000. Antimicrobial susceptibility of Staphylococcus aureus isolated from bovine mastitis in Argentina. J Dairy Sci 83: 1224-1227. doi: $10.3168 / j d s . S 0022-$ 0302(00)74988-5

14. Gonzales RN. 1996. Mastitis y otros factores que afectan a la calidad de la leche. En: Mem Congreso Nacional de Calidad de Leche y Mastitis. Ecuador: Univ Nacional de Río Cuarto.

15. Heringstad B, Klemetsdal G, Ruane J. 2000. Selection for mastitis resistance in dairy cattle: a review with focus on the situation in the Nordic countries. Livestock Prod Sci 64: 95-106. doi : 10.1016/S0301-6226(99)00128-1

16. Hernández R, Fernández C, Baptista $P$. 2003. Metodología de la investigación. $3^{a}$. ed. México DF. McGraw Hill-Interamericana. $736 \mathrm{p}$.

17. Karina P. 2011. Estudio de tipos capsulares de Staphylococcus aureus 
aislados de mastitis bovina y respuesta inmune a una bacterina de tipo capsular 5 en bovinos. Tesis de Maestría. Buenos Aires: Universidad Nacional del Litoral. $153 \mathrm{p}$.

18. Mac Faddin JF. 2000. Biochemical tests for identification of medical bacteria. $3^{\text {rd }}$ ed. Baltimore, USA: Lippincott Williams \& Wilkins. $922 \mathrm{p}$.

19. Martínez D, Cruz A, Moreno G. 2013. Resistencia de las bacterias causantes de mastitis bovina frente a los antimicrobianos más frecuentes. Conexión Agropec JDC 3(1): 53-73.

20. [NCCLS] National Committee for Clinical Laboratory Standards. 2002. Performance standards for antimicrobial disk and dilution susceptibility tests for bacteria isolated from animals. Approved Standard. $2^{\text {nd }} e d$. NCCLS 22(6). M31.A2. [Internet]. Disponible en http:/ /demo.nextlab.ir/getattachment/ $45 \mathrm{f} 0 \mathrm{bcc} 90-98$ b 5 - 4705 - a 4 ad 83c4723c6310/CLSI-M31-A2.aspx

21. Nieto D, Berisso R, Demarchi O, Scala E. 2012. Manual de buenas prácticas de ganadería bovina para la agricultura familiar. Buenos Aires, Argentina: FAO. 169 p. [Internet]. Disponible en: http:// www.fao.org/docrep/019/i3055s/ i3055s.pdf

22. Pyörälä S. 2009. Treatment of mastitis during lactation. Ir Vet J 62: 40-44. doi: 10.1186/2046-0481-62-S4-S40.

23. Ramírez Vásquez N, Arroyave Henao O, Cerón Muñoz M, Jaramillo M, Cerón J, Palacio LG. 2011. Factores asociados a mastitis en vacas de la microcuenca lechera del altiplano norte de Antioquia, Colombia. Rev Med Vet 22: 31-42.
24. Ruiz AK, Ponce P, Gomes G, Mota RA, Sampaio E, Lucena ER, et al. 2011. Prevalencia de mastitis bovina subclínica y microorganismos asociados: comparación entre ordeño manual y mecánico, en Pernambuco, Brasil. Rev Salud Anim 33: 57-64.

25. Sacsaquispe R, Ventura G. 2001. Manual de procedimientos bacteriológicos en infecciones intrahospitalarias. Serie de normas técnicas $\mathrm{N}^{\circ} 28$. Lima: Instituto Nacional de Salud. Ministerio de Salud. [Internet]. Disponible en: https:// www.scribd.com/document/138027115/ Manual-Procedimientos-Bacteriologicos-IIH

26. Santiváñez C, Gómez E, Cárdenas L, Escobedo M, Bustinza R, Peña J. 2013. Prevalencia y factores asociados a la mastitis subclínica bovina en los Andes peruanos. Vet Zootec 7: 92-104.

27. [SENASA] Servicio Nacional de Sanidad Agraria. 2015. Atributos del sistema de vigilancia. Mayor coordinación. Lima: SENASA. [Internet]. Disponible en: https://www.senasa.gob.pe/senasa/ atributos/

28. Tikofsky L, Barlow J, Santisteban C, Schukken Y. 2003. A comparison of antimicrobial susceptibility patterns for Staphylococcus aureus in organic and conventional dairy herds. Microb Drug Resist 9: S39-S45. doi: 10.1089/ 107662903322541883

29. Wenz JR, Barrington GM, Garry FB, McSweeney KD, Dinsmore RP, Goodell G, Callan RJ. 2001. Bacteremia associated with naturally occurring acute coliform mastitis in dairy cows. J Am Vet Med Assoc. 219: 976-981.

30. Ziv G 1980. Drug selection and use in mastitis: systemic vs. local therapy. J Am Vet Med Assoc 176: 1109-1115. 